\title{
Research on Co-ordination Method for Task Allocation
}

\author{
Hua $\mathrm{Yu}^{*}$
}

School of Mechanical and Automotive Engineering, Anhui Polytechnic University, Wuhu, 241000, P.R. China

\begin{abstract}
In this paper, a coordination method to solve task allocation problems in manufacturing system is proposed. inspired by the ability of the ants to establish the shortest route from their nests to the food source and back, researchers found out that these ants secrete a substance called 'pheromone' and use its trails as a medium for communicating information among each other. The pheromone-based static and dynamic coordination algorithms for task allocation are also presented. Experimental results demonstrate that this method is effective and therefore it can provide a feasible way for dealing with an actual allocation problem of production tasks in manufacturing areas.
\end{abstract}

Keywords: Coordination algorithm, coordination method, pheromone, task allocation.

\section{INTRODUCTION}

The purpose of task allocation problem is to assign tasks to processors or machine such that some objective function can be maximized or minimized subject to some constraints [1]. There are many methods to solve this kind problem, such as graph theory techniques [2], branch-and-bound [3], genetic algorithm [4], tabu search [5], simulated annealing [6], and neural network [7].

A graph matching approach was used to solve the heterogeneous system problem under various constraints by Shen and Tsai [8]. An allocation technique was used to solve the incapacitated homogeneous systems by Sarje and Sagar [9]. Sinclair et al., [10] and Billionnet [11] use branch-and-bound algorithms to solve the task allocation problem in a heterogeneous multi-processor system with no capacity constraints. A hybrid heuristic was used to solve a task allocation problem in [1]. However, today, for a specific problem, no technique seems to be more appropriate than another. Moreover, the majority of these techniques suffer from two main drawbacks: firstly, their performances are sensitive to initial parameters and need experimental tuning; secondly, their implementation is widely conducted by the considered models or objective, so it is difficult to add a new constraint or to consider a new model [12].

That is why we decided to solve the allocation problem by using a quite innovative approach, pheromone-based coordination method, which is a relatively novel meta-heuristic based on the indirect communication of a colony of artificial ants mediated by pheromone trails with the collaboration and knowledge-sharing mechanism during their food-seeking process. The ant colony optimization (ACO) was the most recently developed. ACO has a powerful capacity to find out good solutions to combinatorial optimization problems.
Therefore, ACO has been widely applied to solving various combinatorial optimization problems such as task allocation problem [13], job-shop scheduling problem (JSP) [14], traveling salesman problem (TSP) [15], etc. This paper uses an indirect coordination approach based on pheromone to optimize global performance for task allocation.

The remainder of this paper is organized as follows. In section 2, the pheromone-based coordination mechanism is given. In section 3, the pheromone-based static and dynamic coordination algorithms are discussed. An example is presented to demonstrate the validity of this approach to control task allocation in section 4. Finally, some concluding remarks are given in section 5 .

\section{PHEROMONE-BASED COORDINATION MECHA- NISM}

The Ant Colony Optimization techniques has emerged recently as a relatively novel meta-heuristic for hard combinational optimization problems. It is designed to simulate the ability of ant colonies to determine shortest paths to food. Although individual ants posses few capabilities, their operation as a colony is capable of complex behavior.

Real ants can indirectly communicate by pheromone information without using visual cues and are capable of finding the shortest path between food sources and their nests. The ant deposits pheromone on the trail while walking, and the other ants follow the pheromone trails with some probability which are proportioned to the density of the pheromone. The more ants walk on a trail, the more pheromone is deposited on it and more and more ants follow the trail. Through this mechanism, ants will eventually find the shortest path. Artificial ants imitate the behavior of real ants forage of food, but can solve much more complicated problems than real ants can. A search algorithm with such concept is called Ant Colony Optimization. Fig. (1) shows how the real ants find the shortest path [16]. 


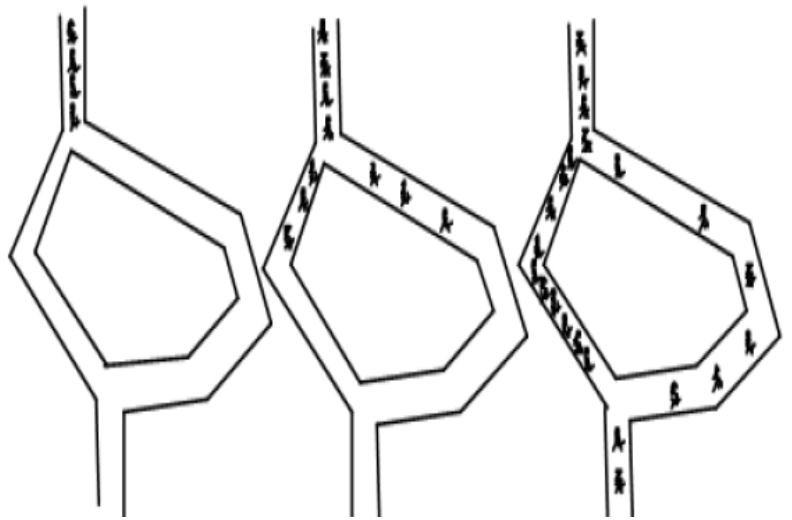

Fig. (1). How real ants find a shortest path.

A further research indicates by Gao et al., [17] that the behavior model for ant colony to choose path for food can be presented in (1).

$p_{i}=\frac{\left(k+c_{i}\right)^{n}}{\sum_{j=1}^{m}\left(k+c_{j}\right)^{n}}$

Where, $p_{i}$ is the probability for ant to choose path $i$ for food; $c_{i}$ and $c_{j}$ are the quantity of pheromone on path $i$ and $j$ respectively; $k$ is attractive degree for no pheromone paths; $n$ is a nonlinear coefficient, and we set $\mathrm{n}$ to 1 here.

\section{PHEROMONE-BASED DYNAMIC COORDINA- TION}

\subsection{Pheromone-based Static Coordination Algorithms}

In ants' food foraging process, pheromone is the basic information element and resides in the route it associates. The function of pheromone is to indicate the attraction of its resident route. The prominent characteristic of pheromone is simplicity [17]. Therefore, the task allocation can be looked as ants' food foraging process. Generally speaking, a task can be finished by several routes; in order to make each route have a chance to be selected, therefore in the beginning, each route that has ability to finish the given task was set an initial value, as shown in (2).

$$
\rho[i][j]= \begin{cases}c_{0} & \text { if route } i \text { can process work piece } j \\ 0 & \text { otherwise }\end{cases}
$$

When a task needs to select a route to process their work pieces, the work piece firstly perceives the pheromone quantum of each feasible route and selects a process route by route's selecting probability, which can be obtained from (1), as shown in (3).

$$
p(i)=\frac{\rho[i][j]}{\sum_{i=1}^{m} \rho[i][j]}
$$

where $i$ represents one process route and $j$ represents the work piece of the task; $p(i)$ is the selecting probability of process route $i$ and $\rho[i][j]$ is route i's pheromone to work piece $j$.

After that the selecting algorithm generates a random number and chooses a process route for processing work piece $\mathrm{j}$ according to calculated selecting probability, then the selected route will get an award, and the "effective pheromone" would be increased. Meanwhile, the involving machine's available processing time on the selected process route will gradually be reduced. Therefore, a pheromone penalty function $m(t)(0<m(t)<\lambda(c))$ are used to reduce the strength of the pheromone in order to reduce the probability of its being selected again, as shown in (4).

$$
\rho[i][j]=\rho[i][j]+\lambda(c)-m(t)
$$

where, $c$ is the total processing cost of the selected route; $\lambda(c)$ is the increased pheromone quantum, and the increment is inversely proportional to the processing cost. $m(t)$ is a decreasing function on the least available time machine of the process route.

When the available time in a certain machine of the process route is less than the corresponding process time processed by this machine for the next work piece, and the pheromone value of the process route automatically becomes zero. Particularly, when a machine's available time is zero, the pheromone value is set to zero for all process routes associated with the machine.

\subsection{Pheromone-based Dynamic Coordination for New Order/Job Arrival}

There are many sources of uncertainty in real-world manufacturing system, which trigger disturbance events in dynamic adaptation manufacturing system control. Generally speaking, there are two types of disturbances, which are resource-related disturbance and source-related disturbance [18].

Source-related disturbance refers to the disturbance caused by the changes in production orders, including new order/job arrival and existing order/job cancellation.

Resource-related disturbance refers to the disturbance caused by unreliability coming from resources (machines) in the shop, including machine breakdown and machine recovery.

The Source-related disturbance is considered in this paper.

The new tasks involved a larger scope, and it may refer to different types of work piece set. Here, for a brief description of the pheromone-based dynamic coordination process for task allocation, assuming that the new task only contains one type of work piece. Only the new feature information of processing tasks is known. Fig. (2) shows the progress of dynamic coordination by using pheromone-like technique when a machine has a malfunction at time $\mathrm{t}=0$. And the specific dynamic coordination mechanism works as follows: 


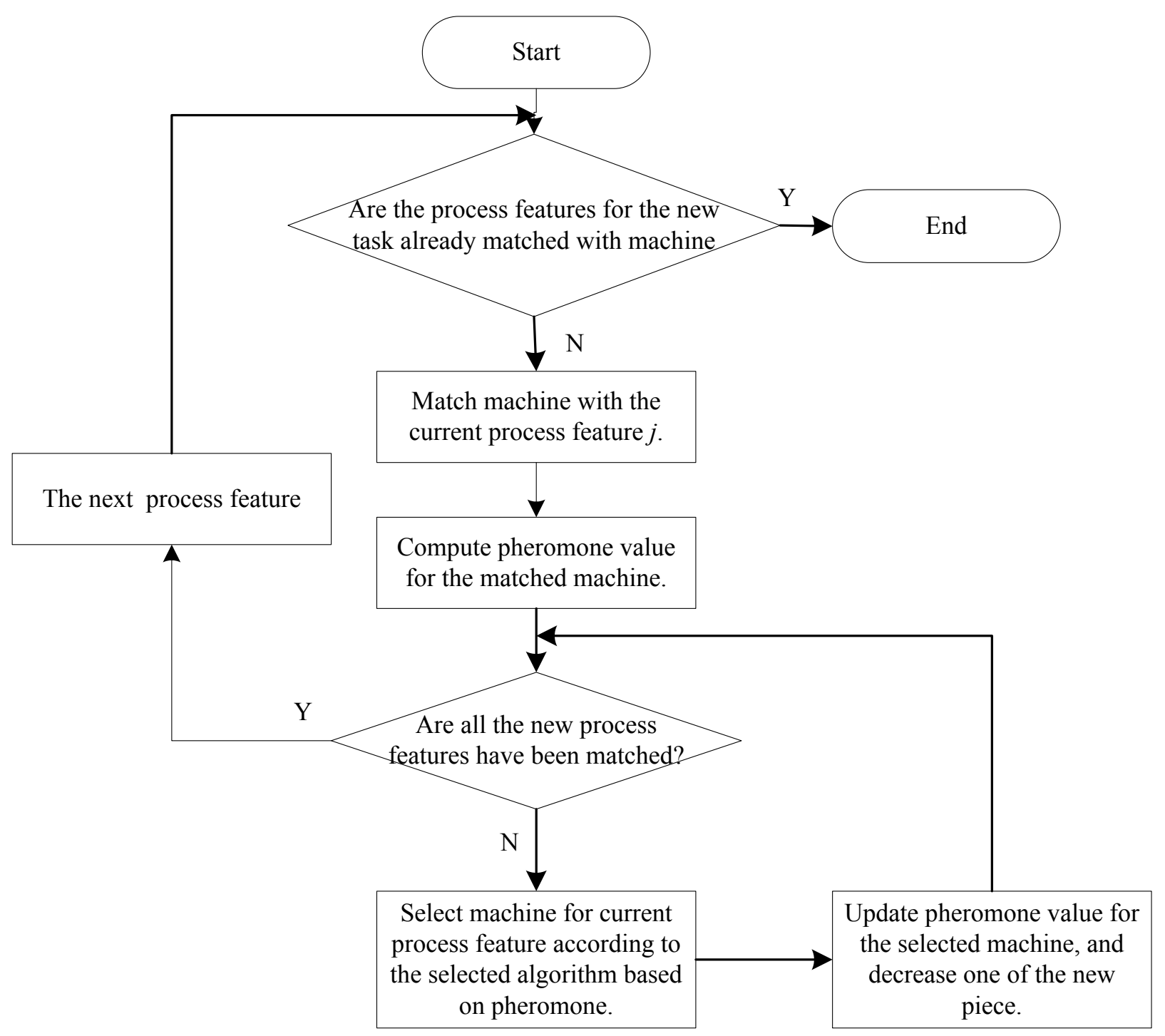

Fig. (2). Pheromone-based dynamic coordination for new order/job arrival.

(1) Select a matching process capability for each processing features of new task.

(2) Add the pheromone value left on the machine in each alternative processing route, as shown in (5).

$q_{i}=\sum_{r=1}^{R} a_{r i}$

The differences in pheromone values calculated by the formula (5) indicate that the ability for each individual machine to process task is different. Under such condition, select a process route by route's selecting probability, which can be obtained from (6).

$p_{i}=\frac{q_{i}}{\sum q_{i}}$

(3) Update the pheromone value on each machine.

(4) Select machine for each processing feature of this kind job until all processing features are selected by the corresponding ability machine.
(5) Count the number for each processing feature on the available machine. Those machine which process relatively more work pieces self-organize into a main virtual manufacturing cell. Meanwhile, those machines which process relatively small work pieces self-organize into one or more vice virtual manufacturing cells.

(6) After the task is completed, the dynamic primary and vice virtual manufacturing cell will automatically disappear.

\section{EXAMPLE AND RESULT}

There are 9 machines in a manufacturing plant. In a certain time, there are three tasks needs to be processed, and the production information for these tasks is shown in Table (1).

Algorithmic parameters are selected as follows: $\mathrm{c} 0=80$, $\alpha(\mathrm{c})=9500 / \mathrm{c}, \mathrm{P}\left(\alpha\left(t_{\min }\right), A(c)\right)=\alpha\left(t_{\min }\right) * \alpha(c)$, where $\alpha\left(t_{\min }\right.$ )$=\mathrm{D} /\left(\mathrm{D}+8 t_{\min }\right), \mathrm{D}=1500$.

Solved by pheromone-based algorithm, the following results are obtained: $\mathrm{n} 11=19 ; \mathrm{n} 12=24 ; \mathrm{n} 13=22 ; \mathrm{n} 21=19$; 
Table 1. Production information for tasks.

\begin{tabular}{|c|c|c|c|c|c|c|}
\hline Task & Quantity & Routes & \multicolumn{4}{|c|}{ Machine/Processing Time /Cost Per Time Unit } \\
\hline \multirow{2}{*}{$P_{1}$} & \multirow{2}{*}{65} & $1-1$ & $1 / 12 / 4$ & $3 / 18 / 3$ & $4 / 15 / 2$ & $6 / 15 / 8$ \\
\hline & & $1-3$ & $8 / 15 / 6$ & $2 / 12 / 5$ & & \\
\hline \multirow[t]{2}{*}{$\mathrm{P}_{2}$} & \multirow[t]{2}{*}{90} & $2-2$ & $4 / 8 / 2$ & $3 / 15 / 3$ & $9 / 10 / 2$ & $7 / 15 / 12$ \\
\hline & & $2-3$ & $1 / 8 / 4$ & $4 / 8 / 2$ & $6 / 5 / 8$ & \\
\hline \multirow[b]{2}{*}{$\mathrm{P}_{3}$} & \multirow[b]{2}{*}{80} & $3-1$ & $8 / 6 / 6$ & $9 / 10 / 2$ & $7 / 25 / 12$ & \\
\hline & & $3-2$ & $6 / 12 / 8$ & $1 / 10 / 4$ & $3 / 5 / 3$ & \\
\hline
\end{tabular}

Table 2. The dynamic coordination results for new task.

\begin{tabular}{|c|c|c|c|c|}
\hline Machine ID & Machine Type & Turning /Number & Milling /Number & Drilling/Number \\
\hline 1 & turning machine & 42 & & \\
\hline 3 & machining center & & 16 & 45 \\
\hline 9 & drilling machine & & & 10 \\
\hline
\end{tabular}

$\mathrm{n} 22=30 ; \mathrm{n} 23=41 ; \mathrm{n} 31=31 ; \mathrm{n} 32=24 ; \mathrm{n} 33=25 . n \mathrm{ij}=\mathrm{N}(\mathrm{i}=1,2,3$; $\mathrm{j}=1,2,3)$ denotes that $\mathrm{N}$ work pieces of task Pi will be processed on the route of Pi-Rj ( $\mathrm{N}$ is less than or equal to the batch size of Pi). For example, n11=19 denotes that eighteen work pieces of $\mathrm{P} 1$ are planned to be processed on the P1-R1 route.

Assume that a new task arrives at time t0, and then task contains 55 work pieces. The task needs to be processed by turning, drilling and milling procedure respectively.

Table (2) shows the dynamic coordination result for new task arrival. Among them, machines M1, M2 and M3 are formed into a main manufacturing cell, and M3, M5 and M9 are formed into a vice manufacturing cell.

The tasks can be well allocated between different alternative machines by using our proposed coordination method. The relatively low-cost and alternative machines can be well selected to process the new tasks, but also take into account the full use of the idle machines' processing capabilities. Therefore, the manufacturing system can be fully utilized as soon as possible. A new main virtual dynamic manufacturing cell can be formed to deal with the primary task. On the other hand, the formed vice manufacturing cell is use to assist the main manufacturing cell. Therefore, some tasks can be well allocated by coordinating among several manufacturing cells, which not only takes into account the machine's loading rate, but also achieve the equalization of each machine in manufacturing system.

\section{CONCLUSION}

Inspired by social insects, a pheromone-based dynamic coordination for task allocation is proposed in this paper. According to that, a pheromone-based task allocation algorithm is proposed. Based on pheromone technique, an example is given and experimental results confirm that the proposed approach is feasible for dynamic coordination task allocations.

The application of pheromone-based coordination approach in manufacturing system is focused on basic task allocation problems and is only tested by using computer, therefore, it is still preliminary and further research work is needed. Ongoing and future work will consider following issues: (1) applying the proposed coordination approach to real industrial cases. (2) The set of optimization techniques and optimization criteria will be expanded. (3) Improving the proposed coordination algorithms so as to enhance system performance. 


\section{CONFLICT OF INTEREST}

The author confirms that this article content has no conflict of interest.

\section{ACKNOWLEDGEMENTS}

The authors would like to thank the referees, the editors and the anonymous reviewers for their helpful comments and constructive suggestions.

\section{REFERENCES}

[1] W. H. Chen, and C. S. Lin, "A hybrid heuristic to solve a task allocation problem”, Computers \& Operations Research, vol. 24, pp. 287-303, 2000.

[2] H. Stone, "Multiprocessor scheduling the aid of network flow algorithms", IEEE Transactions on Software Engineering, vol. 3, no. 1, pp. 85-93, 1997.

[3] H. J. Park, and B. Kim, "An optimal scheduling algorithm for minimizing the computing period of cyclic synchronous tasks on multiprocessors", The Journal of Systems and Software, vol. 56, pp. 213-229, 2001.

[4] J. Aguilar, and E.Gelenbe, "Task assignment and transaction clustering heuristics for distributed systems", Information Sciences, vol. 97, no. 2, pp. 199-219, 1997.

[5] S.C.S. Porto, and C.C. Ribeiro, "A tabu search approach to task scheduling on heterogeneous processors under precedence constraints", International Journal of High-Speed Computing, vol. 7, no. 2, pp. 45-71, 1995.

[6] A. A. Elsadek, and B. E. Wells, "A heuristic model for task allocation inheterogeneous distributed computing systems", The International Journal of Computers and their Applications, vol. 6, no. 1, 1999.

[7] J. Aguilar, and E. Gelenbe, "Task assignment and transaction clustering heuristics for distributed systems", Information Sciences, vol. 97, no. 2, pp. 199-219, 1997.
[8] C. C. Shen, and W. H Tsai, "A graph matching approach to optimal task assignment in distributed computing systems using a minimax criterion", IEEE Transactions on Computers, vol. 34, pp. 197-203, 1985.

[9] A. K. Sarje, and G. Sagar, "Heuristic model for task allocation in distributed computer systems", Computers and Digital Techniques, vol. 138, pp. 313-318, 1991.

[10] J. B Sinclair, "Efficient computation of optimal assignments for distributed tasks", Journal of Parallel and Distributed Computing, vol. 4, pp. 342-362, 1987.

[11] A. Billionnet, M. C. Costa, and A. Sutter, "An efficient algorithm for a task allocation problem", Journal of the Association for Computing Machinery Quarterly, vol. 29, pp. 147-150, 1984.

[12] Pierre-Emmanuel Hladik, Hadrien Cambazard, Anne-Marie De' planche, and Narendra Jussien, "Solving a real-time allocation problem with constraint programming", The Journal of Systems and Software, vol. 81, pp. 132-149, 2008.

[13] S. H. Liu, and Y. Zhang, "Multi-robot task allocation based on particle swarm and ant colony optimal," Journal of Northeast Normal University, vol. 41, no. 4, pp. 68-72, 2009.

[14] S. Kumar, and C. S. P. Rao, "Application of ant colony, genetic algorithm and data mining-based techniques for scheduling", Robotics and Computer-Integrated Manufacturing, vol. 25, pp. 901908, 2009.

[15] Z. C. S. S. Hlaing, and M. A. Khine, "Solving traveling salesman problem by using improved ant colony optimization algorithm", International Journal of Information and Education Technology, vol. 1, no. 5, pp. 404-409, 2011.

[16] L. Min, and J. Yant, "A shortest path routing based on ant algorithm," Journal of Communication and Computer, vol. 2, no. 3, pp. 189-196, 2005.

[17] Q. L. Gao, X. Luo, and S. Z. Yang, "Stigmergic cooperation mechanism for shop floor control system", International Journal of Advanced Manufacturing Technology, vol. 25, pp. 743-753, 2005

[18] W. Xiang, and H. P. Lee, "Ant colony intelligence in multi-agent dynamic manufacturing scheduling", Engineering Applications of Artificial Intelligence, vol. 21, pp.73-85, 2008.

Received: September 22, 2014

Revised: November 05, 2014

Accepted: November 06, 2014

(C) Hua Yu; Licensee Bentham Open.

This is an open access article licensed under the terms of the Creative Commons Attribution Non-Commercial License (http://creativecommons.org/licenses/by-nc/3.0/) which permits unrestricted, non-commercial use, distribution and reproduction in any medium, provided the work is properly cited. 